\title{
In vitro susceptibility of $\beta$-lactamase-producing carbapenem-resistant Enterobacteriaceae (CRE) to eravacycline
}

\author{
Yunliang Zhang, Xiaoyan Lin and Karen Bush \\ Eravacycline is a novel, fully synthetic fluorocycline antibiotic of the tetracycline class being developed for the treatment of \\ complicated urinary tract infections and complicated intra-abdominal infections. Eravacycline has activity against many key \\ Gram-negative pathogens, including Enterobacteriaceae resistant to carbapenems, cephalosporins, fluoroquinolones and \\ $\beta$-lactam $/ \boldsymbol{\beta}$-lactamase inhibitor combinations, including strains that are multidrug-resistant. Carbapenem-resistant \\ Enterobacteriaceae (CRE) isolates from 2010 to $2013(n=110)$ were characterized for carbapenemase genes by PCR and \\ sequencing. MICs for eravacycline, tetracycline, tigecycline, amikacin, imipenem, ceftazidime, cefotaxime and levofloxacin were \\ determined in broth microdilution assays. All isolates produced at least one carbapenemase, most frequently KPC-3. Nine \\ isolates produced both a KPC serine carbapenemase and a metallo- $\beta$-lactamase, NDM-1 $(n=1)$ or VIM-1 $(n=8)$. The 110 \\ isolates were highly resistant to all the $\beta$-lactams tested and to levofloxacin, and had $\mathrm{MIC}_{50} / \mathrm{MIC}_{90}$ values in the intermediate \\ range for tetracycline and amikacin. $\mathrm{MIC}_{50} / \mathrm{MIC}_{90}$ values for eravacycline were $1 / 2 \mu \mathrm{g} \mathrm{ml}^{-1}$ compared with $2 / 2 \mu \mathrm{g} \mathrm{ml}^{-1}$ for \\ tigecycline. Eravacycline MICs were often twofold lower than for tigecycline, with $64 \%$ of the eravacycline MICs $<2 \mu \mathrm{gl} \mathrm{ml}^{-1}$ as \\ compared with $<4 \%$ of tigecycline MICs. Overall, eravacycline demonstrated the lowest cumulative MICs against this panel of \\ recent CRE and may have the potential to treat infections caused by CRE.
}

The Journal of Antibiotics (2016) 69, 600-604; doi:10.1038/ja.2016.73; published online 29 June 2016

\section{INTRODUCTION}

Carbapenem-resistant Enterobacteriaceae (CRE) represents one of the most urgent antibiotic resistance threats as defined by the United States Centers for Disease Control and Prevention. ${ }^{1}$ Infections caused by CRE are responsible for increased morbidity and mortality, with death rates as high as $65 \%$ in special populations. ${ }^{2,3}$ Therapeutic options remain limited for patients infected with antibiotic-resistant Gram-negative infections, ${ }^{4}$ although several new agents have recently been approved, or are in late stage clinical development to treat these patients. ${ }^{5}$ Many of these newly approved or investigational antibiotics have emerged from the previously successful $\beta$-lactam class, either as single agents or as combinations in which a $\beta$-lactamase inhibitor is added to a cephalosporin, carbapenem or monobactam. ${ }^{6,7}$ However, most of the new $\beta$-lactamase inhibitor combinations will provide effective therapy against only those CRE that produce serine carbapenemases such as those in the KPC family, and are not useful against organisms that produce metallo- $\beta$-lactamases (MBLs). ${ }^{8}$ Infectious disease authorities have emphasized the need for new agents in classes other than the $\beta$-lactams in order to lessen the pressure to select for $\beta$-lactam resistance, or to provide antibacterial agents with a different set of adverse events in clinical settings. ${ }^{9}$ As a result, new molecules from other antibacterial classes are also being developed to treat CRE infections. ${ }^{6,7}$
Eravacycline (Figure 1) is a novel, fully synthetic fluorocycline antibiotic of the tetracycline class. Eravacycline has potent in vitro activity against antibiotic-resistant bacteria identified as urgent or serious threats by Centers for Disease Control and Infection: CRE, methicillin-resistant Staphylococcus aureus, extended-spectrum B-lactamase-producing Enterobacteriaceae, vancomycin-resistant enterococci and multidrug-resistant Acinetobacter. ${ }^{10,11}$ It evades many resistance mechanisms seen for tetracycline, minocycline and doxycycline, with no loss of antibacterial activity in the presence of tetracycline ribosomal protection proteins and most tetracyclinespecific efflux pumps. ${ }^{11}$ In isogenic sets of Escherichia coli, eravacycline exhibited elevated MICs in the presence of the tet(A) (but not the tet (B)) efflux pump, and in a strain with overexpression of tet $(\mathrm{X})$, a tetracycline inactivating monooxygenase. ${ }^{12}$ It has demonstrated in vitro antibacterial activity against biofilms formed by uropathogenic E. coli. ${ }^{13}$ Eravacycline has also demonstrated efficacious activity in murine septicemia, and neutropenic mouse lung and thigh model infections. ${ }^{14}$

Eravacycline has completed a Phase 2 trial for the treatment of complicated intra-abdominal infections ${ }^{14}$ and two Phase 3 trials, one for the treatment of complicated intra-abdominal infections and one for the treatment of complicated urinary tract infections (https:// clinicaltrials.gov/). Because eravacycline has the potential to be dosed

Molecular and Cellular Biochemistry Department, Indiana University, Bloomington, IN, USA

Correspondence: Professor K Bush, Molecular and Cellular Biochemistry Department, Indiana University, 212 S. Hawthorne, Bloomington, IN 47405, USA. E-mail: karbush@indiana.edu

This article is dedicated to the fond memory of the late Professor Lester Mitscher, a great scholar, teacher and Emeritus Editor of The Journal of Antibiotics. Received 18 March 2016; revised 25 May 2016; accepted 26 May 2016; published online 29 June 2016 
both parenterally and orally, it is possible that it might be useful in both inpatient and outpatient settings where resistance may be endemic. $^{15}$

Several collections of contemporary Gram-negative bacteria have been studied to determine the in vitro activity of eravacycline and comparative antibacterial agents that have been useful as therapeutic agents to treat infections caused by Gram-negative pathogens. ${ }^{12,16,17}$ An additional set of CRE isolates with well-characterized antibiotic resistance profiles, dating from 2010 to 2013, has recently been collected from central Indiana health-care centers. ${ }^{18,19}$ In the current study, eravacycline and seven comparator drugs including two other tetracyclines, three $\beta$-lactams, the aminoglycoside amikacin and the fluoroquinolone levofloxacin were tested for antimicrobial activity against 110 characterized multidrug-resistant CRE isolates from Indiana. ${ }^{18-20}$ These multidrug-resistant isolates produced primarily KPC carbapenemases, with nine isolates originally identified as producers of MBLs. Comparative MICs and susceptibility profiles determined for all the drugs have shown that eravacycline demonstrates potent antimicrobial activity against these isolates.

\section{MATERIALS AND METHODS}

\section{Antibacterial agents}

With the exception of eravacycline which was furnished by Tetraphase Pharmaceuticals (Watertown, MA, USA), all of the antibacterial agents were obtained from U.S. Pharmacopeial Convention (USP, Rockville, MD, USA). The ranges of concentrations for testing were eravacycline $\left(0.25-16 \mu \mathrm{g} \mathrm{ml}^{-1}\right)$, tetracycline $\left(1-64 \mu \mathrm{g} \mathrm{ml}^{-1}\right)$, tigecycline $\left(0.5-32 \mu \mathrm{g} \mathrm{ml}^{-1}\right)$, amikacin (1$\left.64 \mu \mathrm{g} \mathrm{ml}^{-1}\right)$, imipenem $\left(1-64 \mu \mathrm{g} \mathrm{ml}^{-1}\right)$, ceftazidime $\left(0.5-32 \mu \mathrm{g} \mathrm{ml}^{-1}\right)$, cefotaxime $\left(0.12-8 \mu \mathrm{g} \mathrm{ml}^{-1}\right.$ ) and levofloxacin (either $0.25-16$ or $1-64 \mu \mathrm{g} \mathrm{ml}^{-1}$ ).<smiles>CN(C)[C@H]1C(O)=C(C(N)=O)C(=O)[C@]2(O)C(O)=C3C(=O)c4c(O)c(NC(=O)CN5CCCC5)cc(F)c4C[C@H]3C[C@H]12</smiles>

Figure 1 Structure of eravacycline.

\section{Bacterial strains}

CRE isolates were collected from 2010 through $2013(n=110)$ by two central clinical microbiology laboratories that service 14 central Indiana Health Care Centers and two large urban hospitals. ${ }^{18,19,21}$ Isolates were originally speciated and tested for susceptibility using a Vitek 2 automated system (BioMérieux Inc., St Louis, MO, USA). ${ }^{18}$ Isolates with elevated carbapenem MICs were tested for carbapenemase activity using the modified Hodge test. ${ }^{18,22}$ Before broth dilution susceptibility testing, all isolates were subsequently tested for carbapenemase activity using the Carba NP test with imipenem as the substrate. ${ }^{23}$

\section{$\beta$-Lactamase characterization}

For those isolates that gave a positive carbapenemase test, $\beta$-lactamase genes were identified using the PCR. Plasmid DNA, and genomic DNA for Serratia marcescens, was purified by GenElute Bacterial Genomic DNA Kit (Sigma Life Science, St Louis, MO, USA). Genomic DNA from S. marcescens was screened for the presence of $b a_{S M E}, 24,25$ and plasmids were screened for the presence of bla $a_{C T X-M}, b l a_{O X A}, b l a_{S H V}, b l a_{T E M}, b l a_{K P C}, b l a_{I M P}, b l a_{N D M}$ and $b l a_{V I M}$ by PCR amplification with published primer sets. ${ }^{26-30}$ Sequencing of the PCR products that tested positive for bla genes was performed using the BigDye $3.1 \mathrm{kit}$ (Applied Biosystems, Foster City, CA, USA) and analyzed by BLAST (http:// blast.ncbi.nlm.nih.gov/).

\section{In vitro susceptibility test methods}

MICs were determined by broth microdilution according to Clinical Laboratory Standards Institute (CLSI) guidelines, ${ }^{31}$ using E. coli ATCC 25922 as the quality control strain. The most recent FDA (tigecycline) or CLSI interpretive criteria were applied. ${ }^{22}$ Testing was conducted in duplicate on separate days, with additional replicates assayed if MIC values were non-identical for the first pair of results. The median MIC was reported.

\section{RESULTS}

The bacterial isolates included in the study and their putative $\beta$-lactamases are listed in Table 1. Klebsiella pneumoniae was the most common species $(n=96,87.3 \%)$, followed by E. coli $(n=6,5.5 \%)$, S. marcescens $(n=6,5.5 \%)$ and Enterobacter cloacae $(n=2,1.8 \%)$. All isolates produced a serine carbapenemase, with 107 isolates harboring a KPC carbapenemase (16 KPC-2 and $91 \mathrm{KPC}-3$ variants) and three $S$. marcescens isolates producing the chromosomal SME-1 serine carbapenemase. MBLs produced together with KPC-3 appeared in

Table 1 CRE isolates used for susceptibility testing

\begin{tabular}{|c|c|c|c|c|}
\hline Organism & Serine carbapenemase ${ }^{a}$ & $M B L^{\mathrm{b}}$ & $\mathrm{n}$ & Other $\beta$-lactamase families $(\mathrm{n})^{\mathrm{c}}$ \\
\hline Enterobacter cloacae & KPC-3 & VIM-1 & 2 & TEM (+ AmpC) (1), OXA+SHV+TEM (+ AmpC) (1) \\
\hline \multirow[t]{3}{*}{ Escherichia coli } & KPC-2 & $-{ }^{d}$ & 1 & TEM (1) \\
\hline & KPC-3 & - & 4 & TEM+CTX-M-15 (1), SHV+TEM+CTX-M-15 (1), OXA+SHV+TEM+CTX-M-15 (2) \\
\hline & KPC-3 & VIM-1 & 1 & SHV+TEM (1) \\
\hline \multirow[t]{5}{*}{ Klebsiella pneumoniae } & KPC-2 & - & 15 & TEM (1), SHV+TEM (13), SHV+TEM+OXA (1) \\
\hline & KPC-3 & - & 75 & OXA (1), SHV (4), TEM (19) \\
\hline & & & & OXA+TEM (2), SHV+TEM (37), SHV+CTX-M-15 (2) SHV+TEM+CTX-M-15 (3), OXA+SHV+TEM (2) \\
\hline & KPC-3 & NDM-1 & 1 & $-^{d}$ \\
\hline & KPC-3 & VIM-1 & 5 & TEM (2). TEM+SHV (2), TEM+SHV+OXA (1) \\
\hline \multirow[t]{2}{*}{ Serratia marcescens } & KPC-3 & - & 3 & TEM (+ AmpC) (1), SHV+TEM (+AmpC) (2) \\
\hline & SME & - & 3 & SHV (+ AmpC) (3) \\
\hline
\end{tabular}

aCarbapenemases were initially detected using a modified Hodge test and confirmed by the Carba NP test. All carbapenemase genes were detected by PCR, with sequencing of the full gene used to identify the carbapenemase.

betallo- $\beta$-lactamase.

${ }^{c}$ Additional $\beta$-lactamases were detected by PCR, with full or partial sequencing of individual genes. Most enzymes were identified only according to enzyme families. For $E$. cloacae and S. marcescens a chromosomal AmpC was assumed to be present

dNone detected. 
Table 2 Susceptibility data for eravacycline and comparative agents tested against characterized CRE isolates

\begin{tabular}{|c|c|c|c|c|c|}
\hline \multirow[b]{2}{*}{ Organisms } & \multirow[b]{2}{*}{ Antibiotic } & \multicolumn{3}{|c|}{ MIC in $\mu g \mathrm{ml}^{-1}$ (interpretive criteria) } & \multirow[b]{2}{*}{$\%$ Susceptible $^{a}(n)$} \\
\hline & & Range & $M I C_{50}$ & $M I C_{90}$ & \\
\hline \multirow[t]{8}{*}{ All carbapenem-resistant Enterobacteriaceae $(n=110)$} & Eravacycline & $0.5-4$ & 1 & 2 & $N A^{b}$ \\
\hline & Tetracycline & $4->64$ & $8(\mathrm{l})$ & $8(\mathrm{I})$ & $35.5(39)$ \\
\hline & Tigecycline & $1-4$ & $2(\mathrm{~S})$ & $2(S)$ & $91.8(101)$ \\
\hline & Amikacin & $2->64$ & $32(1)$ & $32(\mathrm{I})$ & $34.5(38)$ \\
\hline & Imipenem & $4->64$ & $8(R)$ & $32(\mathrm{R})$ & $0(0)$ \\
\hline & Ceftazidime & $1->32$ & $>32(\mathrm{R})$ & $>32(\mathrm{R})$ & $1.8(2)$ \\
\hline & Cefotaxime & $1->8$ & $>8(\mathrm{R})$ & $>8(\mathrm{R})$ & $0.9(1)$ \\
\hline & Levofloxacin & $\leqslant 0.25->16$ & $>16(\mathrm{R})$ & $>16(\mathrm{R})$ & $2.7(3)$ \\
\hline \multirow[t]{8}{*}{ KPC-producing K. pneumoniae $(n=96)$} & Eravacycline & $0.5-4$ & 1 & 2 & NA \\
\hline & Tetracycline & $4-8$ & $8(\mathrm{l})$ & $8(\mathrm{l})$ & $37.5(36)$ \\
\hline & Tigecycline & $1-4$ & $2(S)$ & $2(S)$ & $94.8(91)$ \\
\hline & Amikacin & $2->64$ & $32(I)$ & $32(\mathrm{I})$ & $29.2(28)$ \\
\hline & Imipenem & $4->64$ & $8(R)$ & $32(R)$ & $0(0)$ \\
\hline & Ceftazidime & $>32$ & $>32(\mathrm{R})$ & $>32(\mathrm{R})$ & $0(0)$ \\
\hline & Cefotaxime & $>8$ & $>8(\mathrm{R})$ & $>8(\mathrm{R})$ & $0(0)$ \\
\hline & Levofloxacin & $2->16$ & $>16(\mathrm{R})$ & $>16(\mathrm{R})$ & $1.0(1)$ \\
\hline
\end{tabular}

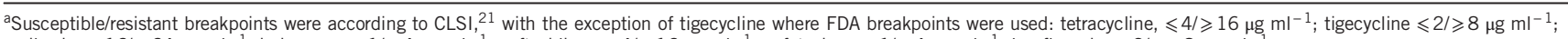
amikacin, $\leqslant 16 / \geqslant 64 \mu \mathrm{g} \mathrm{ml}^{-1}$; imipenem, $\leqslant 1 / \geqslant 4 \mu \mathrm{g} \mathrm{ml}^{-1}$; ceftazidime, $\leqslant 4 / \geqslant 16 \mu \mathrm{g} \mathrm{ml}^{-1}$; cefotaxime, $\leqslant 1 / \geqslant 4 \mu \mathrm{g} \mathrm{ml}-1$; levofloxacin, $\leqslant 2 / \geqslant \geq 8 \mu \mathrm{g} \mathrm{ml}$. .

${ }^{b} N A$, Not applicable. No breakpoints have been established.

Table 3 MIC distributions of eravacycline and comparator agents against 110 CRE isolates

\begin{tabular}{|c|c|c|c|c|c|c|c|c|c|c|c|c|}
\hline \multirow[b]{2}{*}{ Antibiotic } & \multicolumn{12}{|c|}{ MIC in $\mu g \mathrm{ml}^{-1}$} \\
\hline & $\leqslant 0.5$ & 1 & 2 & 4 & 8 & $>8$ & 16 & $>16$ & 32 & $>32$ & 64 & $>64$ \\
\hline Eravacycline & 2 & 68 & 36 & 4 & & & & & & & & \\
\hline Tetracycline & & & $2^{a}$ & 37 & 66 & & & & 3 & & & 2 \\
\hline Tigecycline & & 4 & 97 & 9 & & & & & & & & \\
\hline Amikacin & & & 3 & 5 & 3 & & 27 & & 62 & & 6 & 4 \\
\hline Imipenem & & & & 4 & 58 & & 25 & & 12 & & 6 & 5 \\
\hline Ceftazidime & & $1^{a}$ & $1^{\mathrm{a}}$ & & & & & & & 108 & & \\
\hline Cefotaxime & & $1^{\mathrm{a}}$ & $1^{\mathrm{a}}$ & & & 108 & & & & & & \\
\hline Levofloxacin & $2^{a}$ & & 1 & 2 & 5 & & 4 & 96 & & & & \\
\hline
\end{tabular}

${ }^{\mathrm{a}} \mathrm{S}$. marcescens producing SME-1.

E. cloacae (VIM-1, $n=2)$, E. coli (VIM-1, $n=1)$ and K. pneumoniae (NDM-1, $n=1$; VIM-1, $n=5$ ).

Susceptibility data for eravacycline and comparative antibiotics are summarized in Table 2. When data were analyzed according to $\mathrm{MIC}_{50}$ and $\mathrm{MIC}_{90}$ values, $\mathrm{MIC}_{50} / \mathrm{MIC}_{90}$ values did not vary for any of the drugs according to the two organism sets, that is, all the CRE or only KPC-producing $K$. pneumoniae. The full CRE collection exhibited $<3 \%$ susceptibility to the carbapenem imipenem, the cephalosporins ceftazidime and cefotaxime, and the fluoroquinolone levofloxacin. Two S. marcescens that produced the serine carbapenemase SME-1 were not resistant to the cephalosporins (MICs $1-2 \mu \mathrm{g} \mathrm{ml}^{-1}$ ) and levofloxacin (MICs $\leqslant 0.25 \mu \mathrm{g} \mathrm{ml}^{-1}$ ), but remained highly resistant to imipenem with MICs $>64 \mu \mathrm{g} \mathrm{ml}^{-1}$ (Table 3). None of the KPCproducing $K$. pneumoniae strains tested as susceptible to any of the $\beta$-lactams that were tested. Tetracycline and amikacin exhibited similar susceptibility profiles for all the CRE, with $35.5 \%$ (39/110) and 34.5\% $(38 / 110)$ of the strains susceptible; $60 \%(66 / 110)$ and $56.4 \%(62 / 110)$ of the isolates tested in the intermediate range for tetracycline and amikacin, respectively. A lower percentage of $K$. pneumoniae CRE isolates $(29.2 \%$ or $28 / 96)$ was susceptible to amikacin compared with the full complement of CRE isolates, whereas tetracycline susceptibility in K. pneumoniae (37.5\% or $36 / 96)$ was slightly higher than for the composite set of isolates that were tested.

Eravacycline had an $\mathrm{MIC}_{50}$ of $1 \mu \mathrm{g} \mathrm{ml}^{-1}$, the lowest of all the agents tested, and one doubling dilution lower than tigecycline $\left(\mathrm{MIC}_{50}\right.$ equal to $2 \mu \mathrm{g} \mathrm{ml}^{-1}$ ). Modal MICs corresponded to the $\mathrm{MIC}_{50}$ values of 1 and $2 \mu \mathrm{g} \mathrm{ml}^{-1}$ for eravacycline and tigecycline, respectively. For both agents, $\mathrm{MIC}_{90}$ values were $2 \mu \mathrm{g} \mathrm{ml}^{-1}$, the lowest $\mathrm{MIC}_{90}$ values of all the agents tested. Tigecycline MICs were in the intermediate range $\left(4 \mu \mathrm{g} \mathrm{ml}^{-1}\right)$ for $8.2 \%(9 / 110)$ of the isolates. For 73 isolates $(66.4 \%)$, tigecycline MICs were twofold higher than MICs for eravacycline. At MICs $\leqslant 1 \mu \mathrm{g} \mathrm{ml}^{-1}$, eravacycline inhibited growth of $63.6 \%$ (70/110) of the isolates, compared with $3.6 \%$ (4/110) for tigecycline. Four isolates had eravacycline MICs of $4 \mu \mathrm{g} \mathrm{ml}^{-1}$; of these, three had equivalent tigecycline MICs and one had a tigecycline MIC of $2 \mu \mathrm{g} \mathrm{ml}^{-1}$. Two of the isolates with eravacycline and tigecycline MICs of $4 \mu \mathrm{g} \mathrm{ml}^{-1}$ were K. pneumoniae isolates that produced both a KPC-3 and a VIM-1 carbapenemase. Overall, the ranges of eravacycline and tigecycline MICs spanned only a fourfold concentration range of $1-4 \mu \mathrm{g} \mathrm{ml}^{-1}$ for $98 \%$ of the isolates, in contrast to tetracycline with an MIC range of 4$>64 \mu \mathrm{g} \mathrm{ml}^{-1}$ for the CRE isolates, indicating that common 
tetracycline resistance mechanisms do not seem to be greatly affecting the antibacterial activity of the two newer tetracycline analogs.

\section{DISCUSSION}

Eravacycline demonstrated the most potent inhibitory concentrations of all the antibiotics that were tested against the set of multidrugresistant CRE isolates. Not only were these isolates highly resistant to $\beta$-lactam antibiotics and levofloxacin, but they also demonstrated low susceptibility to tetracycline and amikacin. Although the $\mathrm{MIC}_{50}$ value $\left(1 \mu \mathrm{g} \mathrm{ml}^{-1}\right)$ for eravacycline in this study was $2-8$-fold higher than reported in other studies of E. coli and K. pneumoniae isolates, ${ }^{10,11,32}$ the $\mathrm{MIC}_{90}$ was identical to the corresponding data in the Sutcliffe study. ${ }^{11}$ The K. pneumoniae and fluoroquinolone-resistant E. coli isolates in earlier studies were also more sensitive to tigecycline that had an $\mathrm{MIC}_{50}$ of $0.5-1 \mu \mathrm{g} \mathrm{ml}^{-111,32}$ compared with an $\mathrm{MIC}_{50}$ of $2 \mu \mathrm{g} \mathrm{ml}^{-1}$ in this study. The Indiana set of CRE demonstrated greater antibiotic resistance than the isolates in the previous studies that were only $10-33 \%$ resistant to the expanded-spectrum cephalosporin ceftriaxone and $0.4-13 \%$ resistant to meropenem, ${ }^{10,32}$ compared with $100 \%$ resistance to ceftazidime, cefotaxime and imipenem in this set of isolates, possibly explaining the greater sensitivity to eravacycline in earlier studies.

Tigecycline is an accepted therapeutic agent that is currently utilized for treating CRE in some health-care centers. However, eravacycline was usually twofold more potent than tigecycline against the majority of CRE isolates in the current study. The unique organism collection that was tested included a KPC carbapenemase in $>97 \%$ of the strains, usually with at least one other $\beta$-lactamase, and, in some cases, as many as four other $\beta$-lactamases. KPC carbapenemases represent the most common carbapenemase in North America and frequently appear in multidrug-resistant pathogens. $^{33,34}$ Nine isolates co-produced a KPC serine $\beta$-lactamase together with an MBL that utilizes zinc at its active site to hydrolyze $\beta$-lactams. Although the recently approved $\beta$-lactamase inhibitor combination ceftazidime-avibactam has useful activity against many KPC-producing CRE, it is ineffective against CRE that produce MBLs such as VIM-1, or the NDM-1 enzyme that is increasing in prevalence. ${ }^{34,35}$ Thus, eravacycline that demonstrated MICs of $2 \mu \mathrm{g} \mathrm{ml}^{-1}$ or lower against $96 \%$ of the multidrug-resistant CRE isolates, including those with MBLs, may provide a non- $\beta$-lactam alternative to treating infections caused by these difficult-to-treat organisms. In addition, the potential development of an orally active formulation of eravacycline would be valuable in the antibacterial armamentarium, as all the other agents available for treatment of CRE are parenteral agents. Based on the in vitro data presented, eravacycline has the potential to be considered for further development for possible treatment of infections caused by CRE.

\section{CONFLICT OF INTEREST}

The authors declare no conflict of interest.

\section{ACKNOWLEDGEMENTS}

We acknowledge the support of Tetraphase Pharmaceuticals to conduct this study.

1 Centers for Disease Control and Prevention. Antibiotic Resistance Threats in the United States (United States Department of Health and Human Services, Atlanta, GA, USA, 2013).

2 Drekonja, D. M. et al. Challenges in the management of infections due to carbapenemresistant Enterobacteriaceae. Infect. Control Hosp. Epidemiol. 35, 437-439 (2014).
3 Satlin, M. J., Jenkins, S. G. \& Walsh, T. J. The global challenge of carbapenem-resistant Enterobacteriaceae in transplant recipients and patients with hematologic malignancies. Clin. Infect. Dis. 58, 1274-1283 (2014).

4 Bassetti, M. \& Righi, E. New antibiotics and antimicrobial combination therapy for the treatment of gram-negative bacterial infections. Curr. Opin. Crit. Care 21 , 402-411 (2015).

5 Bush, K. Investigational agents for the treatment of Gram-negative bacterial infections: a reality check. ACS Infect. Dis. 1, 509-511 (2015).

6 Amin, A. N. \& Deruelle, D. Healthcare-associated infections, infection control and the potential of new antibiotics in development in the USA. Future Microbiol. 10, 1049-1062 (2015).

7 Page, M. G. P. \& Bush, K. Discovery and development of new antibacterial agents targeting Gram-negative bacteria in the era of pandrug resistance: is the future promising? Curr. Opin. Pharmacol. 18, 91-97 (2014).

8 Bush, K. A resurgence of $\beta$-lactamase inhibitor combinations effective against multidrugresistant Gram-negative pathogens. Intl. J. Antimicrob. Agents 46, 483-493 (2015).

9 Infectious Diseases Society of Americaet al. Combating antimicrobial resistance: policy recommendations to save lives. Clin. Infect. Dis. 52(suppl. 5), S397-S428 (2011).

10 Abdallah, M. et al. Activity of eravacycline against Enterobacteriaceae and Acinetobacter baumannii, including multidrug-resistant isolates, from New York City. Antimicrob. Agents Chemother. 59, 1802-1805 (2015).

11 Sutcliffe, J. A., O'Brien, W., Fyfe, C. \& Grossman, T. H. Antibacterial activity of eravacycline (TP-434), a novel fluorocycline, against hospital and community pathogens. Antimicrob. Agents Chemother. 57, 5548-5558 (2013).

12 Grossman, T. H. et al. Target- and resistance-based mechanistic studies with TP-434, a novel fluorocycline antibiotic. Antimicrob. Agents Chemother. 56, 2559-2564 (2012).

13 Grossman, T. H., O'Brien, W., Kerstein, K. O. \& Sutcliffe, J. A. Eravacycline (TP-434) is active in vitro against biofilms formed by uropathogenic Escherichia coli. Antimicrob. Agents Chemother. 59, 2446-2449 (2015).

14 Grossman, T. H., Murphy, T. M., Slee, A. M., Lofland, D. \& Sutcliffe, J. A. Eravacycline (TP-434) is efficacious in animal models of infection. Antimicrob. Agents Chemother. 59, 2567-2571 (2015).

15 Solomkin, J. S. et al. Phase 2, randomized, double-blind study of the efficacy and safety of two dose regimens of eravacycline versus ertapenem for adult community-acquired complicated intra-abdominal infections. Antimicrob. Agents Chemother. 58, 1847-1854 (2014).

16 Livermore, D. M., Mushtaq, S., Warner, M. \& Woodford, N. Activity of eravacycline against carbapenemase-producing Enterobacteriaceae and Acinetobacter baumannii. Abstract F-769. in 55th Interscience Conference on Antimicrobial Agents and Chemotherapy (San Diego, CA, USA, 2015).

17 Zhanel, G. G. et al. Activity of eravacycline and comparators against 3,174 pathogens isolated from Canadian hospitals: CANWARD 2014. Abstract F-771. in 55th Interscience Conference on Antimicrobial Agents and Chemotherapy (San Diego, CA, USA, 2015).

18 Denys, G. A. et al. Changing epidemiology and molecular characterization of carbapenem-resistant Enterobacteriaceae (CRE) in Indiana Health Care Centers (HCCs) over a 3-year period. Abstract \# C2-1445. in: 53rd Conf. Antimicrobial Agents and Chemotherapy (Denver, CO, USA, 2013)

19 Bush, K. et al. Detection systems for carbapenemase gene identification should include the SME serine carbapenemase. Intl. J. Antimicrob. Agents 41, 1-4 (2013).

20 Dunn, M. et al. KPC-2 and VIM carbapenemases decreased in carbapenem-resistant Enterobacteriaceae in Indiana health care centers from 2009 to 2013. in 54th Interscience Conference on Antimicrobial Agents \& Chemotherapy (Washington, DC, USA, 2014).

21 Kashikar, A. et al. Occurrence of VIM and NDM carbapenemases in Indiana health care centers from 2009 to 2013. Abstract C-1095. in 55th Interscience Conference on Antimicrobial Agents and Chemotherapy (San Diego, CA, USA, 2015).

$22 \mathrm{CLSI}$ Performance Standards for Antimicrobial Susceptibility Testing. CLSI supplement M100S, 26th edn (Clinical and Laboratory Standards Institute, Wayne, PA, 2016)

23 Nordmann, P., Poirel, L. \& Dortet, L. Rapid detection of carbapenemase-producing Enterobacteriaceae. Emerg. Infect. Dis. 18, 1503-1507 (2012).

24 Fairfax, M. R. et al. Detection of 2 SME-1 carbapenemase-producing Serratia marcescens in Detroit. Diagn. Microbiol. Infect. Dis. 71, 325-326 (2011).

25 Queenan, A. M. et al. SME-type carbapenem-hydrolyzing class A $\beta$-lactamases from geographically diverse Serratia marcescens strains. Antimicrob. Agents Chemother. 44, 3035-3039 (2000).

26 Yigit, H. et al. Novel carbapenem-hydrolyzing $\beta$-lactamase, KPC-1, from a carbapenemresistant strain of Klebsiella pneumoniae. Antimicrob. Agents Chemother. 45 1151-1161 (2001).

27 Lolans, K., Queenan, A. M., Bush, K., Sahud, A. \& Quinn, J. P. First nosocomial outbreak of Pseudomonas aeruginosa producing an integron-borne metallo- $\beta$-lactamase (VIM-2) in the United States. Antimicrob. Agents Chemother. 49, 3538-3540 (2005).

28 Queenan, A. M. et al. SME-3, a novel member of the Serratia marcescens SME family of carbapenem-hydrolyzing $\beta$-lactamases. Antimicrob. Agents Chemother. 50, 3485-3487 (2006).

29 Sidjabat, H. et al. Molecular epidemiology of CTX-M-producing Escherichia coli isolates at a tertiary medical center in western Pennsylvania. Antimicrob Agents Chemother. 53, 4733-4739 (2009).

30 Poirel, L., Walsh, T. R., Cuvillier, V. \& Nordmann, P. Multiplex PCR for detection of acquired carbapenemase genes. Diagn. Microbiol. Infect. Dis. 70, 119-123 (2011). 
31 CLSI. M07-A9: Methods for Dilution Antimicrobial Susceptibility Tests for Bacteria That Grow Aerobically; Approved Standard. 9th edn (Clinical and Laboratory Standards Institute, Wayne, PA, 2013).

32 Johnson, J. R., Porter, S. B., Johnston, B. D. \& Thuras, P. Activity of eravacycline against Escherichia coli clinical isolates collected from U.S. veterans in 2011 in relation to coresistance phenotype and sequence type 131 genotype. Antimicrob. Agents Chemother 60, 1888-1891 (2015).

33 Castanheira, M., Farrell, S. E., Krause, K. M., Jones, R. N. \& Sader, H. S. Contemporary diversity of $\beta$-lactamases among Enterobacteriaceae in the nine U.S. census regions and ceftazidime-avibactam activity tested against isolates producing the most prevalent $\beta$-lactamase groups. Antimicrob Agents Chemother. 58, 833-838 (2014).

34 Centers for Disease Control \& Prevention. Notes from the field: New Delhi metallo- $\beta$ lactamase-producing Escherichia coli associated with endoscopic retrograde cholangiopancreatography - Illinois, 2013. Morbid. Mortal. Week Rep. 62, 1051 (2014).

$35 \mathrm{Li}, \mathrm{H}$. et al. In vitro susceptibility of characterized $\beta$-lactamase-producing strains tested with avibactam combinations. Antimicrob Agents Chemother. 59, 1789-1793 (2015). 